\section{Reply to S. Yuan et al}

Yuan and $\mathrm{Su}^{1}$ highlight several important questions for additional clarification and discussion. Regarding the staging of patients with natural killer (NK)/T-cell lymphoma with active disease who were treated, we report that even patients with advanced stage (eg, bone marrow) disease were included. ${ }^{2}$ Most of the patients with NK/T-cell non-Hodgkin lymphoma (NHL) had experienced failure of both chemotherapy and radiation therapy. The study did not dictate the type of therapy that patients received before cytotoxic T-cell (CTL) therapy. For example, patient 1054 was treated with radiotherapy and cyclophosphamide, doxorubicin, vincristine, and prednisone before outcomes with the new chemotherapy regimen consisting of dexamethasone, methotrexate, ifosfamide, $\mathrm{L}$-asparaginase, and etoposide were published. Yuan and $\mathrm{Su}^{1}$ queried the phenotype in the patients with NK/T-cell lymphoma who did not respond to latent membrane protein-specific cytotoxic T-lymphocyte (LMP-CTL) therapy, and in both cases the residual malignant populations were EBER-positive $\mathrm{CD} 3{ }^{+} \mathrm{CD} 56^{+} \mathrm{T}$ cells.

As highlighted in the discussion, there are multiple reasons why LMP-CTL therapy might not clear NK/T-cell NHL in vivo. Yuan and $\mathrm{Su}^{1}$ proposed several potential mechanisms for failure, and current studies are looking at strategies to overcome the immune evasion mechanisms employed by the tumor cells, as well as combining CTL therapies with chemotherapy regimens and targeted therapies. We agree that an important reason for immune escapes is antigen loss, a problem that may be overcome by epitope spreading, which may explain why lack of epitope spreading in some of our patients correlated with lack of tumor response.

The authors also question whether LMP-CTLs attack nonmalignant $\mathrm{B}$ cells in vivo. We have never observed loss of normal B cells, but there are reports that lymphoblastoid cell lines can activate T cells specific for nonviral autoantigens such as phosphopeptides. These phosphopeptides may only be expressed by activated B cells, and we have not evaluated if activated B cells are selectively eliminated.

Yuan and $\mathrm{Su}^{1}$ also requested additional clarification regarding the cohort treated in remission. All five patients who received LMPCTL therapy as adjuvant therapy for NK/T-cell NHL maintained a continued complete response of more than 2 years. Of these five patients, four received radiotherapy, while the fifth was treated with systemic chemotherapy only.

We hope that our answers to the questions raised by Yuan and $\mathrm{Su}^{1}$ will aid in the understanding of the therapeutic mechanisms of LMP-CTL therapy for the treatment of patients with refractory Epstein-Barr virus-positive NK/T-cell lymphomas.

\section{Catherine M. Bollard and Helen E. Heslop}

Center for Cell and Gene Therapy, Dan L. Duncan Cancer Center, Baylor College of Medicine; The Methodist Hospital; and Texas Children's Hospital, Houston, TX

\section{Cliona M. Rooney}

Center for Cell and Gene Therapy, Dan L. Duncan Cancer Center, Baylor College of Medicine; and Texas Children's Hospital, Houston, TX

\section{ACKNOWLEDGMENT}

H.E.H. is supported by a Dan L. Duncan Chair.

\section{AUTHORS' DISCLOSURES OF POTENTIAL CONFLICTS OF INTEREST}

Disclosures provided by the authors are available with this article at www.jco.org.

\section{REFERENCES}

1. Yuan S, Su H: Latent membrane protein-specific cytotoxic T lymphocytes: New hope for patients with natural killer/T-cell lymphoma? 32:2819-2820, 2014

2. Bollard CM, Gottschalk S, Torrano V, et al: Sustained complete responses in patients with lymphoma receiving autologous cytotoxic T lymphocytes targeting Epstein-Barr virus latent membrane proteins. J Clin Oncol 32:798-808, 2014

DOI: 10.1200/JCO.2014.56.8469; published online ahead of print at www.jco.org on July 21, 2014 


\section{AUTHORS' DISCLOSURES OF POTENTIAL CONFLICTS OF INTEREST}

Reply to S. Yuan et al

The following represents disclosure information provided by authors of this manuscript. All relationships are considered compensated. Relationships are self-held unless noted. I = Immediate Family Member, Inst = My Institution. For a detailed description of the disclosure categories, or for more information about ASCO's conflict of interest policy, please refer to the Author Disclosure Declaration and the Disclosures of Potential Conflicts of Interest section in Information for Contributors.

Catherine M. Bollard

Honoraria: Cellmedica (I)

Consulting or Advisory Role: Cellmedica (I)

Helen E. Heslop

Honoraria: Cellmedica (I)
Consulting or Advisory Role: Cellmedica (I)

Research Funding: Celgene (Inst)

Patents, Royalties, Other Intellectual Property: Cell Medica (I)

Cliona M. Rooney

Consulting or Advisory Role: Cellmedica (I) 